PROCEEDINGS OF THE AMERICAN MATHEMATICAL SOCIETY

Volume 127, Number 3, March 1999, Pages 805-811

S 0002-9939(99)04570-0

\title{
THE BERGMAN KERNEL FUNCTION: EXPLICIT FORMULAS AND ZEROES
}

\author{
HAROLD P. BOAS, SIQI FU, AND EMIL J. STRAUBE
}

(Communicated by Steven R. Bell)

\begin{abstract}
We show how to compute the Bergman kernel functions of some special domains in a simple way. As an application of the explicit formulas, we show that the Bergman kernel functions of some convex domains, for instance the domain in $\mathbb{C}^{3}$ defined by the inequality $\left|z_{1}\right|+\left|z_{2}\right|+\left|z_{3}\right|<1$, have zeroes.
\end{abstract}

\section{INTRODUCTION}

This note has two themes. The first is that one can obtain useful formulas for the Bergman kernel function in special cases with minimal computational effort. The second is that one can find from the explicit formulas examples of simple convex domains whose Bergman kernel functions have zeroes.

Recently there has been renewed interest [6], [8], [9], [13] in explicit formulas for the Bergman kernel function, especially in connection with the asymptotic behavior of the kernel near weakly pseudoconvex boundary points. We will show how, even without writing down an infinite series, one can easily obtain an explicit formula for the Bergman kernel function of, for example, the domain defined by the inequality $\left\|z_{1}\right\|^{2 / p_{1}}+\cdots+\left\|z_{n}\right\|^{2 / p_{n}}<1$, where each $z_{j}$ is a vector in some $\mathbb{C}^{m_{j}}$, and each $p_{j}$ is a positive integer.

Finding a simple class of domains whose Bergman kernel functions are zero-free has been an open problem ever since Lu Qi-Keng [12] raised the question in connection with the existence of Bergman representative coordinates. The first author recently discovered [3] that the Bergman kernel of a generic strongly pseudoconvex domain does have zeroes, but the possibility was left open that all convex domains might have zero-free Bergman kernel functions. We will show that the Bergman kernel function of the domain in $\mathbb{C}^{3}$ defined by the inequality $\left|z_{1}\right|+\left|z_{2}\right|+\left|z_{3}\right|<1$ does have zeroes. Consequently, when $n \geq 3$, there exists a smooth, bounded, strongly convex Reinhardt domain in $\mathbb{C}^{n}$, even with real analytic boundary, whose Bergman kernel function has zeroes.

\section{Explicit Formulas For the Bergman Kernel}

The Bergman kernel function $K(z, w)$ of a domain $\Omega$ in $\mathbb{C}^{n}$ is the reproducing kernel for the space of square-integrable holomorphic functions, that is, $f(z)=$ $\int_{\Omega} f(w) K(z, w) d V_{w}$ when $f$ is a square-integrable holomorphic function in $\Omega$. We

Received by the editors June 30, 1997.

1991 Mathematics Subject Classification. Primary 32H10.

This research was supported in part by NSF grant number DMS 9500916 . 
use three basic principles to obtain new Bergman kernel functions from old ones. The principles of deflation and inflation relate kernel functions of domains in different dimensions. On the other hand, the well known principle of folding relates kernel functions of domains in the same dimension.

2.1. Deflation. Let $\Omega$ be a bounded domain in $\mathbb{C}^{n}$ defined by an inequality of the form $\varphi(z)<1$, where $\varphi$ is a continuous, nonnegative function in a neighborhood of the closure of $\Omega$. Let $K_{1}$ denote the Bergman kernel function of the bounded domain $\Omega_{1}$ in $\mathbb{C}^{n+2}$ defined by the inequality $\varphi(z)+\left|\zeta_{1}\right|^{2 / p}+\left|\zeta_{2}\right|^{2 / q}<1$, where $p$ and $q$ are positive real numbers. Similarly, let $K_{2}$ denote the Bergman kernel function of the bounded domain $\Omega_{2}$ in $\mathbb{C}^{n+1}$ defined by the inequality $\varphi(z)+|\zeta|^{2 /(p+q)}<1$. Then

$$
\pi K_{2}(z, 0, w, 0)=\frac{\pi^{2} \Gamma(p+1) \Gamma(q+1)}{\Gamma(p+q+1)} K_{1}(z, 0,0, w, 0,0) .
$$

For example, we will show below in $\S 3.1$ that the Bergman kernel function $K\left(z_{1}, z_{2}, w_{1}, w_{2}\right)$ of the nonconvex domain $\left\{\left(z_{1}, z_{2}\right):\left|z_{1}\right|+\left|z_{2}\right|^{2 / 4}<1\right\}$ in $\mathbb{C}^{2}$ has zeroes when $z_{2}=w_{2}=0$. Taking $n=1$ and $p=q=2$ in (1) shows that the Bergman kernel function of the convex domain $\left\{\left(z_{1}, z_{2}, z_{3}\right):\left|z_{1}\right|+\left|z_{2}\right|+\left|z_{3}\right|<1\right\}$ in $\mathbb{C}^{3}$ also has zeroes.

The deflation identity (1) holds because both sides represent the (unique) holomorphic reproducing kernel function for the holomorphic functions on $\Omega$ that are square-integrable with respect to the weight function $(1-\varphi)^{p+q}$. (Consult [11] for a systematic study of weighted Bergman projections.) To see this, observe that a holomorphic function $h$ on $\Omega$ can be regarded as a holomorphic function on both $\Omega_{1}$ and $\Omega_{2}$ by extending it to be independent of the extra variables. The reproducing property of the Bergman kernel function on $\Omega_{2}$ implies that

$$
h(z)=\int_{\Omega_{2}} h(w) K_{2}(z, 0, w, \eta) d V_{w, \eta} .
$$

In the domain $\Omega_{2}$, the fiber over a point $w$ in $\Omega$ is a one-dimensional disc of radius $(1-\varphi(w))^{(p+q) / 2}$. Integrating with respect to $\eta$ and using the mean-value property of harmonic functions shows that (2) reduces to

$$
h(z)=\int_{\Omega} h(w)(1-\varphi(w))^{(p+q)} \pi K_{2}(z, 0, w, 0) d V_{w} .
$$

The subaveraging property of $\left|K_{2}(z, 0, w, 0)\right|^{2}$ implies that $K_{2}(z, 0, w, 0)$ is squareintegrable with respect to the weight $(1-\varphi)^{p+q}$, so the left-hand side of (1) is the reproducing kernel for the holomorphic functions on $\Omega$ that are square-integrable with respect to the weight $(1-\varphi)^{p+q}$.

The verification of the reproducing property of the right-hand side of (1) differs only in that the fiber over a point in $\Omega$ is two-dimensional, and one needs the volume of the region in $\mathbb{C}^{2}$ defined by the inequality $\left|\zeta_{1}\right|^{2 / p}+\left|\zeta_{2}\right|^{2 / q}<R$. A routine calculation in polar coordinates shows that this volume equals

$$
\pi^{2} \Gamma(p+1) \Gamma(q+1) R^{p+q} / \Gamma(p+q+1),
$$

which completes the proof of the deflation identity (1). 
2.2. Inflation. Let $\Omega$ be a bounded complete Hartogs domain in $\mathbb{C}^{n+1}$ defined by an inequality of the form $|\zeta|^{2}<\varphi(z)$, where $\zeta \in \mathbb{C}, z \in \mathbb{C}^{n}$, and $\varphi$ is a bounded, positive, continuous function on the interior of some bounded domain in $\mathbb{C}^{n}$. Due to the circular symmetry in the one-dimensional variable, there is a function $L(z, w, t)$ such that the Bergman kernel function $K(z, \zeta, w, \eta)$ for $\Omega$ can be written in the form $L(z, w, \zeta \bar{\eta})$. We can inflate $\Omega$ into a domain $\widetilde{\Omega}$ in $C^{n+m}$ defined by the inequality $\|Z\|^{2}<\varphi(z)$, where $Z$ is a vector variable in $\mathbb{C}^{m}$ with Euclidean norm $\|Z\|$. We will show that the Bergman kernel function $\widetilde{K}(z, Z, w, W)$ of $\widetilde{\Omega}$ is given by the relation

$$
\widetilde{K}(z, Z, w, W)=\left.\frac{1}{\pi^{m-1}} \frac{\partial^{m-1}}{\partial t^{m-1}} L(z, w, t)\right|_{t=\langle Z, W\rangle},
$$

where $\langle Z, W\rangle=Z_{1} \bar{W}_{1}+\cdots+Z_{m} \bar{W}_{m}$.

The motivation for this formula is the relation between the Bergman kernel function $\pi^{-1}(1-\zeta \bar{\eta})^{-2}$ for the unit disc in $\mathbb{C}$ and the Bergman kernel function for the unit ball in $\mathbb{C}^{m}$, which is

$$
\left.\frac{1}{\pi^{m}} \frac{\partial^{m-1}}{\partial t^{m-1}} \frac{1}{(1-t)^{2}}\right|_{t=\langle Z, W\rangle}=\frac{m !}{\pi^{m}} \cdot \frac{1}{(1-\langle Z, W\rangle)^{m+1}} .
$$

For an application, recall that when $p$ is a positive real number, the Bergman kernel function of the domain $|z|^{2}+|\zeta|^{2 / p}<1$ in $\mathbb{C}^{2}$ is

$$
\left.\frac{1}{p \pi^{2}} \frac{\partial^{2}}{\partial t^{2}}\left(\frac{1}{(1-t)^{p}-\zeta \bar{\eta}}\right)\right|_{t=z \bar{w}}
$$

where the power $(1-t)^{p}$ denotes the principal branch. (Here is the only place in this note where -implicitly - an infinite series appears. Bergman $[2$, p. 82] computed this kernel by summing a series; although he states that $p$ is integral, his computation is valid for arbitrary positive $p$.) Inflating both variables shows that the Bergman kernel function of the domain $\|z\|^{2}+\|Z\|^{2 / p}<1$ in $\mathbb{C}^{n+m}$ is

$$
\left.\frac{1}{p \pi^{n+m}} \frac{\partial^{n+m}}{\partial t^{n+1} \partial u^{m-1}}\left(\frac{1}{(1-t)^{p}-u}\right)\right|_{\substack{t=\langle z, w\rangle \\ u=\langle Z, W\rangle}} .
$$

This kernel function was first computed (in a somewhat different form) by Chalmers [4, Theorem 1.1]. Subsequently, D'Angelo used a different method to sum the orthonormal series to compute this kernel for $m=1$ in [5] and for general $m$ in [6]; his formulas are written as finite sums rather than as derivatives.

We prove the inflation formula (3) by verifying the reproducing property. Since $\widetilde{\Omega}$ is invariant under unitary transformations of $\mathbb{C}^{m}$, it suffices to verify (3) when $Z=\left(Z_{1}, 0, \ldots, 0\right)$, in which case we can rewrite

$$
\left.\frac{\partial^{m-1}}{\partial t^{m-1}} L(z, w, t)\right|_{t=\langle Z, W\rangle}=\frac{1}{Z_{1}^{m-1}} \frac{\partial^{m-1}}{\partial \bar{W}_{1}^{m-1}} L\left(z, w, Z_{1} \bar{W}_{1}\right) .
$$

If $h$ is a square-integrable holomorphic function on $\widetilde{\Omega}$, then we find by using the mean-value property in the $m-1$ variables $W_{2}, \ldots, W_{m}$ that

$$
\begin{aligned}
\left.\frac{1}{\pi^{m-1}} \int_{\widetilde{\Omega}} h(w, W) \frac{\partial^{m-1}}{\partial t^{m-1}} L(z, w, t)\right|_{t=Z_{1} \bar{W}_{1}} d V_{w, W}=\frac{1}{(m-1) ! Z_{1}^{m-1}} \\
\times \int_{\Omega} h\left(w, W_{1}\right)\left(\varphi(w)-\left|W_{1}\right|^{2}\right)^{m-1} \frac{\partial^{m-1}}{\partial \bar{W}_{1}^{m-1}} L\left(z, w, Z_{1} \bar{W}_{1}\right) d V_{w, W_{1}} .
\end{aligned}
$$


Observe that $f(z) \mapsto(1-|z|) f^{\prime}(z)$ is continuous in $L^{2}$ on holomorphic functions in the unit disc (this follows by integrating in polar coordinates), so the above calculation implies that the kernel (7) is square-integrable in $\widetilde{\Omega}$. Approximating $h\left(w, W_{1}\right)$ and $L\left(z, w, Z_{1} \bar{W}_{1}\right)$ by polynomials in $W_{1}$ and $\bar{W}_{1}$ respectively shows that no boundary terms arise when we integrate by parts in the $W_{1}$ variable $(m-1)$ times, so the right-hand side of the preceding formula reduces to

$$
\frac{1}{Z_{1}^{m-1}} \int_{\Omega} h\left(w, W_{1}\right) W_{1}^{m-1} L\left(z, w, Z_{1} \bar{W}_{1}\right) d V_{w, W_{1}}=h\left(z, Z_{1}\right),
$$

where the final equality follows from the reproducing property of the Bergman kernel function on $\Omega$. Thus the kernel given by (3) does reproduce holomorphic functions on $\widetilde{\Omega}$.

2.3. Folding. The third method is a well known "useful tool to establish explicit formulas for the Bergman kernel" (Jarnicki and Pflug [10, p. 176]). As in paragraph 2.2 , let $\Omega$ be a bounded complete Hartogs domain in $\mathbb{C}^{n+1}$ defined by an inequality of the form $|\zeta|^{2}<\varphi(z)$. When $p$ is a positive integer, $(z, \zeta) \mapsto\left(z, \zeta^{p}\right)$ is a proper holomorphic mapping of $\Omega$ onto the domain $\Omega_{p}$ in $\mathbb{C}^{n+1}$ defined by the inequality $|\zeta|^{2 / p}<\varphi(z)$. Bell's transformation rule [1] for the Bergman kernel function under proper mappings implies that when $\zeta \bar{\eta} \neq 0$, the Bergman kernel $K$ for $\Omega$ is related to the Bergman kernel $K_{p}$ for $\Omega_{p}$ via

$$
p^{2}(\zeta \bar{\eta})^{p-1} K_{p}\left(z, \zeta^{p}, w, \eta^{p}\right)=\sum_{j=1}^{p} \bar{\omega}^{j} K\left(z, \zeta, w, \omega^{j} \eta\right),
$$

where $\omega$ is a primitive $p$ th root of unity.

For example, we can repeatedly fold formula (6) with $m=1$ to see that when $p_{1}, \ldots, p_{n}$ are positive integers, and $p$ is any positive real number, the domain in $\mathbb{C}^{n+1}$ defined by the inequality $\left|z_{1}\right|^{2 / p_{1}}+\cdots+\left|z_{n}\right|^{2 / p_{n}}+\left|z_{n+1}\right|^{2 / p}<1$ has Bergman kernel $K$ given by the finite sum

$$
\begin{aligned}
K\left(z_{1}^{p_{1}}, \ldots, z_{n}^{p_{n}}, z_{n+1}, w_{1}^{p_{1}}, \ldots, w_{n}^{p_{n}}, w_{n+1}\right)=\frac{1}{p \pi^{n+1}} \prod_{k=1}^{n} \frac{1}{p_{k}^{2}\left(z_{k} \bar{w}_{k}\right)^{p_{k}-1}} \\
\times\left.\sum_{j_{1}=1}^{p_{1}} \cdots \sum_{j_{n}=1}^{p_{n}} \frac{\partial^{n+1}}{\partial t^{n+1}}\left(\frac{\bar{\omega}_{1}^{j_{1}} \ldots \bar{w}_{n}^{j_{n}}}{(1-t)^{p}-z_{n+1} \bar{w}_{n+1}}\right)\right|_{t=\sum_{\ell=1}^{n} z_{\ell} \bar{w}_{\ell} \bar{\omega}_{\ell}^{j_{\ell}}},
\end{aligned}
$$

where each $\omega_{j}$ is a primitive $p_{j}$ th root of unity. Francsics and Hanges [8, Theorem 3] computed this kernel by summing an infinite series. When $p$ is also an integer, this kernel can be obtained by folding the simpler formula (4) for the ball (compare [10, Exercise 6.5]); this special case was first computed by Zinov'ev [15] via series methods.

It should be evident how to inflate (8) via (3) to obtain the Bergman kernel function for the domain defined by the inequality $\left\|z_{1}\right\|^{2 / p_{1}}+\cdots+\left\|z_{n}\right\|^{2 / p_{n}}+\left\|z_{n+1}\right\|^{2 / p}<$ 1 , where each $z_{j}$ is a vector in some $\mathbb{C}^{m_{j}}$. Repeated foldings and inflations easily give the Bergman kernel functions of still more general domains with defining functions like $\left(\left\|z_{1}\right\|^{2 / p_{1}}+\left\|z_{2}\right\|^{2 / p_{2}}\right)^{2 / p}+\left\|z_{3}\right\|^{2 / p_{3}}<1$, the only difficulty in writing the formulas being the choice of a suitable notation (compare $[7, \S 6.2]$ ). 


\section{Zeroes OF THE Bergman KERNEL}

We now deduce from the explicit formulas some consequences about when the Bergman kernel function has zeroes. In particular, the kernel function has zeroes in some simple convex domains, a fact previously unknown.

3.1. Two-dimensional domains. To find the Bergman kernel function $K_{p}$ for the domain $\Omega_{p}$ in $\mathbb{C}^{2}$ defined by the inequality $\left|z_{1}\right|+\left|z_{2}\right|^{2 / p}<1$, we fold Bergman's formula (5), or, equivalently, take $n=1$ and $p_{1}=2$ in (8), leaving $p$ as an arbitrary positive real number. Setting $x:=z_{1} \bar{w}_{1}$ and $y:=z_{2} \bar{w}_{2}$, we have

$$
K_{p}\left(z_{1}^{2}, z_{2}, w_{1}^{2}, w_{2}\right)=\frac{1}{4 p \pi^{2} x} \cdot \frac{\partial^{2}}{\partial x^{2}}\left(\frac{1}{(1-x)^{p}-y}-\frac{1}{(1+x)^{p}-y}\right)
$$

when $x \neq 0$. We will show that the Bergman kernel function $K_{p}$ of $\Omega_{p}$ has zeroes when $p>2$, while the Bergman kernel function $K_{2}$ of $\Omega_{2}$ has no zeroes on the interior of the domain.

Setting $y=0$ in (9), we see that $K_{p}\left(z_{1}^{2}, 0, w_{1}^{2}, 0\right)=0$ if and only if $x \neq 0$ and $(1+x)^{p+2}=(1-x)^{p+2}$. When $y=0$, the point $x$ runs over the unit disc. Since $t \mapsto(1+t) /(1-t)$ maps the unit disc bijectively to the right half-plane, it follows that the equation $[(1+x) /(1-x)]^{p+2}=1$ has a nonzero solution in the unit disc if and only if $p>2$. Hence for $p>2$, the Bergman kernel function $K_{p}$ has zeroes when $z_{2}=0$.

Taking the limit in (9) as $x \rightarrow 0$, we find after a routine calculation that

$$
K_{p}\left(0, z_{2}, 0, w_{2}\right)=\frac{1}{2 \pi^{2}} \cdot \frac{y^{2}\left(p^{2}-3 p+2\right)+4 y\left(p^{2}-1\right)+\left(p^{2}+3 p+2\right)}{(1-y)^{4}} .
$$

This expression is real-valued for real values of $y$, positive for $y=0$, and equal to $-\left(p^{2}-4\right) / \pi^{2}$ at $y=-1$, so if $p>2$, then there is a zero for $y$ in the unit disc. Hence for $p>2$, the Bergman kernel $K_{p}$ has zeroes also when $z_{1}=0$.

Setting $p=2$ in (9), we find after some routine algebra that

$$
K_{2}\left(z_{1}, z_{2}, w_{1}, w_{2}\right)=\frac{2}{\pi^{2}} \cdot \frac{3(1-x-y)\left(1-(x-y)^{2}\right)+8 x y}{\left((1-x-y)^{2}-4 x y\right)^{3}},
$$

where $x=z_{1} \bar{w}_{1}$ and $y=z_{2} \bar{w}_{2}$ as before (compare [10, Example 6.1.9]). We aim to show that this expression has no zeroes when $x$ and $y$ are complex numbers such that $\sqrt{|x|}+\sqrt{|y|}<1$. This constraint implies that $4|x||y|<(1-|x|-|y|)^{2}$, so the numerator of the expression exceeds twice

$$
\begin{aligned}
3(1-|x|-|y|) & \left(1-|x-y|^{2}\right)-2(1-|x|-|y|)^{2} \\
& \geq 3(1-|x|-|y|)^{2}(1+|x-y|)-2(1-|x|-|y|)^{2} \\
\quad & >(1-|x|-|y|)^{2} .
\end{aligned}
$$

Consequently, $K_{2}\left(z_{1}, z_{2}, w_{1}, w_{2}\right)$ has no zeroes on the interior of the domain. However, there are zeroes on the boundary: for instance, $K_{2}(1,0,-1,0)=0$.

Notice that when $p>2$, the case in which we have shown that the Bergman kernel $K_{p}$ has zeroes, the domain $\Omega_{p}$ is not convex. In higher dimensions, the situation changes. 
3.2. Higher-dimensional domains. Using the deflation identity (1), we can identify many convex domains in higher dimensions whose Bergman kernel functions have zeroes. For example, the Bergman kernel function of the convex domain in $\mathbb{C}^{n}$ defined by the inequality $\left|z_{1}\right|+\left|z_{2}\right|+\cdots+\left|z_{n}\right|<1$ has zeroes if and only if the dimension $n \geq 3$. Indeed, we have checked that this Bergman kernel is zero-free when $n=2$. If $n \geq 3$, on the other hand, then we find by (1) that the restriction of the kernel to the subspace where $z_{2}=\cdots=z_{n}=0$ equals a positive constant times the kernel $K_{p}\left(z_{1}, 0, w_{1}, 0\right)$ of $\S 3.1$ with $p=2 n-2$, and we saw in $\S 3.1$ that this kernel has zeroes when $2 n-2>2$.

As another example, we show that the Bergman kernel function $K$ of the convex domain in $\mathbb{C}^{n}$ defined by the inequality $\left|z_{1}\right|+\left|z_{2}\right|^{2}+\left|z_{3}\right|^{2}+\cdots+\left|z_{n}\right|^{2}<1$ has zeroes if and only if the dimension $n \geq 4$. Using deflation, as in the preceding paragraph, shows that the kernel has zeroes when $n \geq 4$, but does not show that the kernel is zero-free when $n \leq 3$. Accordingly, we examine the explicit formula obtained by folding the kernel for the ball:

$$
\begin{aligned}
& K\left(z_{1}^{2}, z_{2}, \ldots, z_{n}, w_{1}^{2}, w_{2}, \ldots, w_{n}\right) \\
& \quad=\frac{n !}{\pi^{n}} \cdot \frac{1}{4 z_{1} \bar{w}_{1}}\left(\frac{1}{(1-\langle z, w\rangle)^{n+1}}-\frac{1}{\left(1+2 z_{1} \bar{w}_{1}-\langle z, w\rangle\right)^{n+1}}\right) .
\end{aligned}
$$

The same argument as in $\S 3.1$, that $t \mapsto(1+t) /(1-t)$ maps the unit disc bijectively to the right half-plane, shows that this kernel function has zeroes in the interior of the domain if and only if $n+1>4$.

By using the tools indicated in $\S 2$, the reader can easily produce more examples of convex domains whose Bergman kernel functions have zeroes. We have given two examples to illustrate the usefulness of the techniques.

The examples just given of convex domains whose Bergman kernel functions have zeroes are nonsmooth domains. We can exhaust such domains by sequences of smooth, strongly convex domains with real-analytic boundaries. By Ramadanov's theorem [14], the Bergman kernels of the approximating domains converge uniformly on compact sets to the Bergman kernel of the limit domain. By Hurwitz's theorem, the Bergman kernel functions of the approximating strongly convex domains cannot all be zero-free. Consequently, when $n \geq 3$, there exist smooth, bounded, strongly convex domains in $\mathbb{C}^{n}$, even with real-analytic boundary, whose Bergman kernel functions have zeroes.

\section{Remarks}

1. The domains in $\mathbb{C}^{n}$ whose Bergman kernels we have shown to have zeroes are convex only when $n \geq 3$. It would be interesting to find a bounded convex domain in dimension $n=2$ whose Bergman kernel function has zeroes.

2. It would be interesting to characterize the set of positive real numbers $p_{1}, p_{2}$, $\ldots, p_{n}$ for which the Bergman kernel function of the domain in $\mathbb{C}^{n}$ defined by the inequality $\left|z_{1}\right|^{2 / p_{1}}+\left|z_{2}\right|^{2 / p_{2}}+\cdots+\left|z_{n}\right|^{2 / p_{n}}<1$ is zero-free.

\section{NOTE ADDED IN PROOF}

Shortly after we emailed P. Pflug about our results, he and E. H. Youssfi were able to find zeroes of the Bergman kernels of the "minimal balls" studied in [13]. Their paper "The Lu Qi-Keng conjecture fails for strongly convex algebraic domains" will appear in Archiv der Mathematik. 


\section{REFERENCES}

[1] Steven R. Bell, The Bergman kernel function and proper holomorphic mappings, Trans. Amer. Math. Soc. 270 (1982), no. 2, 685-691. MR 83i:32033

[2] Stefan Bergmann (Bergman), Zur Theorie von pseudokonformen Abbildungen, Mat. Sb. (N.S.) 1 (43) (1936), no. 1, 79-96.

[3] Harold P. Boas, The Lu Qi-Keng conjecture fails generically, Proc. Amer. Math. Soc. 124 (1996), no. 7, 2021-2027. MR 96i:32024

[4] Bruce L. Chalmers, On boundary behavior of the Bergman kernel function and related domain functionals, Pacific J. Math. 29 (1969), 243-250. MR 40:402

[5] John P. D'Angelo, A note on the Bergman kernel, Duke Math. J. 45 (1978), no. 2, 259-265. MR 57:12906

[6] _ An explicit computation of the Bergman kernel function, J. Geometric Analysis 4 (1994), no. 1, 23-34. MR 95a:32039

[7] G. P. Egorychev, Integral representation and the computation of combinatorial sums, Translations of Mathematical Monographs, vol. 59, American Mathematical Society, 1984. MR 85a:05008

[8] Gábor Francsics and Nicholas Hanges, The Bergman kernel of complex ovals and multivariable hypergeometric functions, J. Funct. Anal. 142 (1996), no. 2, 494-510. MR 97m:32039

[9] __ Asymptotic behavior of the Bergman kernel and hypergeometric functions, Multidimensional Complex Analysis and Partial Differential Equations, Contemporary Mathematics, vol. 205, American Mathematical Society, 1997, pp. 79-92. CMP 97:12

[10] Marek Jarnicki and Peter Pflug, Invariant Distances and Metrics in Complex Analysis, de Gruyter, 1993. MR 94k:32039

[11] Ewa Ligocka, On the Forelli-Rudin construction and weighted Bergman projections, Studia Math. 94 (1989), no. 3, 257-272. MR 90i:32034

[12] Qi-Keng Lu (K. H. Look), On Kaehler manifolds with constant curvature, Chinese Math. 8 (1966), 283-298. MR 34:6806

[13] K. Oeljeklaus, P. Pflug, and E. H. Youssfi, The Bergman kernel of the minimal ball and applications, Ann. Inst. Fourier (Grenoble) 47 (1997), no. 3, 915-928. MR 98d:32028

[14] I. Ramadanov, Sur une propriété de la fonction de Bergman, C. R. Acad. Bulgare Sci. 20 (1967), 759-762. MR 37:1632

[15] B. S. Zinov'ev, On reproducing kernels for multicircular domains of holomorphy, Siberian Math. J. 15 (1974), 24-33. MR 48:11555

Department of Mathematics, Texas A\&M University, College Station, Texas 77843 3368

E-mail address: boas@math.tamu.edu

E-mail address: sfu@math.tamu.edu

Current address, (S. Fu): Department of Mathematics, University of Wyoming, Laramie, Wyoming 82071-3036

E-mail address: straube@math.tamu.edu 